\title{
A bifurcation and symmetry result for critical fractional Laplacian equations involving a perturbation
}

\author{
Jiabin Zuo ${ }^{1,2,3^{*}}$ (D), Mingwei Li ${ }^{4}$, Bomeng Li ${ }^{5}$ and Zhenhua Qiao ${ }^{6}$
}

\section{"Correspondence:}

zuojiabin88@163.com

${ }^{1}$ Faculty of Applied Sciences, Jilin

Engineering Normal University,

Changchun, P.R. China

${ }^{2}$ College of Science, Hohai

University, Nanjing, China

Full list of author information is

available at the end of the article

\section{Springer}

\begin{abstract}
In the present paper, by using the variational and topological methods, we obtain a multiplicity and bifurcation result for the following fractional problems involving critical nonlinearities and a lower order perturbation:

$$
-\mathcal{L}_{k} v=\mu v+|v|^{2_{s}^{*}-2} v+g(x, v) \quad \text { in } \Omega, \quad v=0 \quad \text { in } \mathbb{R}^{N} \backslash \Omega,
$$

where $\Omega$ is an open and bounded domain with Lipschitz boundary, $N>2 s$, with $s \in(0,1), g$ is a lower order perturbation of the critical power $|v|^{2_{s}^{*}-2} v$ and $\mu$ is a positive real parameter, $2_{s}^{*}=\frac{2 N}{N-2 s}$ is the fractional critical Sobolev exponent, while $\mathcal{L}_{K}$ is an integro-differential operator. Precisely, we show that the number of nontrivial solutions for this equation under suitable assumptions is at least twice the multiplicity of the eigenvalue. Our conclusions improve the related results in some respects.
\end{abstract}

MSC: 35A15; 47G20; 35S15; 35Q60

Keywords: Integro-differential operator; Critical nonlinearities; Fractional problems; Variational methods

\section{Introduction}

Recently, fractional nonlocal problems with critical nonlinearities have attracted the interest of mathematicians because of their applications in many fields, such as, flame propagation, water waves, optimization, multiple scattering, and so on. We refer the interested reader to [1-6] and the references therein.

In 1983, Brezis and Nirenberg first proposed a kind of critical problem in [7],

$$
\begin{cases}-\Delta v=\mu \nu+|v|^{2^{*}-2} v & \text { in } \Omega, \\ v=0 & \text { on } \Omega,\end{cases}
$$

which have been widely studied (see for example [8-12]) thanks to relations with problems that arise in differential geometry and physics. Here $\Omega$ is an open and bounded domain, $N>2,2^{*}=\frac{2 N}{N-2}$ is the fractional critical Sobolev exponent. Subsequently, Cerami

(c) The Author(s) 2020. This article is licensed under a Creative Commons Attribution 4.0 International License, which permits use, sharing, adaptation, distribution and reproduction in any medium or format, as long as you give appropriate credit to the original author(s) and the source, provide a link to the Creative Commons licence, and indicate if changes were made. The images or other third party material in this article are included in the article's Creative Commons licence, unless indicated otherwise in a credit line to the material. If material is not included in the article's Creative Commons licence and your intended use is not permitted by statutory regulation or exceeds the permitted use, you will need to obtain permission directly from the copyright holder. To view a copy of this licence, visit http://creativecommons.org/licenses/by/4.0/. 
et al. proved the first multiplicity result of problem (1.1) in [13]. Later, under suitable assumptions of dimension $N$ and parameters, many authors have given the existence and multiplicity of the solution of this kind of problem (for more details see for instance [1417]). In all of the above papers, the classical known existence results are extended to the following nonlocal fractional setting $[5,6,11,18]$ :

$$
\begin{cases}(-\Delta)^{s} v=\mu v+|v|^{2_{s}^{*}-2} v & \text { in } \Omega, \\ v=0 & \text { in } \mathbb{R}^{N} \backslash \Omega,\end{cases}
$$

where $\Omega$ is an open and bounded domain with Lipschitz boundary, $N>2 s$, with $s \in(0,1)$, $\mu$ is a real parameter, $2_{s}^{*}=\frac{2 N}{N-2 s}$ is the fractional critical Sobolev exponent and $-(-\Delta)^{s}$ is the fractional Laplace operator, up to normalization factors, may be defined as follows:

$$
(-\Delta)^{s} v(x):=\int_{\mathbb{R}^{N}}(v(x+y)+v(x-y)-2 v(x))|y|^{-(N+2 s)} d y,
$$

for $x \in \mathbb{R}^{N}$ (see [19] for more details on the fractional Laplacian). Through observation, we find that due to the characteristics of nonlocal problems, the boundary condition of problem (1.2) is a natural counterpart of the Dirichlet boundary condition of problem (1.1). The literature on bifurcated results for nonlocal fractional equations is very interesting, we refer to see $[20,21]$ and the references therein.

Motivated by the above work, the aim of this paper is to investigate the multiplicity of solutions for a class of fractional equations involving critical nonlinearities and a lower order perturbation:

$$
\begin{cases}-\mathcal{L}_{K} v=\mu v+|v|^{2_{s}^{*}-2} v+g(x, v) & \text { in } \Omega, \\ v=0 & \text { in } \mathbb{R}^{N} \backslash \Omega,\end{cases}
$$

where $\mathcal{L}_{K}$ is a integro-differential operator defined as follows:

$$
\mathcal{L}_{K} v(x):=\int_{\mathbb{R}^{N}}(v(x+y)+v(x-y)-2 v(x)) K(y) d y, \quad x \in \mathbb{R}^{N},
$$

where function $K: \mathbb{R}^{N} \backslash\{0\} \rightarrow(0, \infty)$ is a kernel such that

$$
\left\{\begin{array}{l}
\omega K \in L^{1}\left(\mathbb{R}^{N}\right) \\
\text { where } \omega(x)=\min \left\{|x|^{2}, 1\right\} ; \\
\text { there exists } \vartheta>0 \text { such that } K(x) \geq \vartheta|x|^{-(N+2 s)} \\
\text { for any } x \in \mathbb{R}^{N} \backslash\{0\} ; \\
K(x)=K(-x) \\
\text { for any } x \in \mathbb{R}^{N} \backslash\{0\} .
\end{array}\right.
$$

Thanks to taking $K(x)=|x|^{-(N+2 s)}$, we have $\mathcal{L}_{K}=-(-\Delta)^{s}$, so we call the operator $\mathcal{L}_{K}$ is a extension of the fractional Laplacian.

Finally, $g$ is a lower order perturbation of the critical power $|v|^{2 *-2} v$, and it is a Carathéodory function from $\Omega \times \mathbb{R}$ to $\mathbb{R}$ satisfying the following assumptions: 
$\left(g_{1}\right) \sup \{|g(x, t)|$ : a.e. $x \in \Omega, t \in[0, D]\}<+\infty$ for any $D>0$;

$\left(g_{2}\right) \lim _{|t| \rightarrow 0} \frac{g(x, t)}{t}=0$ uniformly for $x \in \Omega$;

$\left(g_{3}\right) \lim _{|t| \rightarrow \infty} \frac{g(x, t)}{|t|^{2 *}-1}=0$ uniformly for $x \in \Omega$;

$\left(g_{4}\right) \quad 0 \leq G(x, t)=\int_{0}^{t} g(x, \widetilde{v}) d \widetilde{v} \leq \frac{1}{2} g(x, t) t+\frac{s-\epsilon}{N}|t|^{2_{s}^{*}}$ a.e. $x \in \Omega, t \in \mathbb{R}$, where $s>\epsilon>0$;

$\left(g_{5}\right) g(x,-t)=-g(x, t)$ for all $x \in \Omega, t \in \mathbb{R}$.

As a typical example for $g$ we can set $g(x, t)=l(x)|t|^{\xi-2} t$, with $l(x) \in L^{\infty}(\Omega), l(x) \geq 0$ a.e. $x \in \Omega$ and $\xi \in\left(2,2_{s}^{*}\right)$.

Obviously, $v \equiv 0$ is a trivial solution of problem $(\mathrm{E})$. Hence, we are only interested in nontrivial solutions. The nontrivial weak solutions of problem $(\mathrm{E})$ is equivalent to the nontrivial solutions of the following problem:

$$
\left\{\begin{array}{l}
\int_{\mathbb{R}^{2 N}}(v(x)-v(y))(\psi(x)-\psi(y)) K(x-y) d x d y \\
\quad=\mu \int_{\Omega} v(x) \psi(x) d x+\int_{\Omega}|v(x)|^{2_{s}^{*}-2} v(x) \psi(x) d x+\int_{\Omega} g(x, v(x)) \psi(x) d x=0, \\
\quad \text { for any } \psi, v \in V_{0},
\end{array}\right.
$$

which represents the Euler-Lagrange equation of the functional $J_{K, \mu}: V_{0} \rightarrow \mathbb{R}$ defined as

$$
\begin{aligned}
J_{K, \mu}(v):= & \frac{1}{2} \int_{\mathbb{R}^{2 N}}|v(x)-v(y)|^{2} K(x-y) d x d y-\mu \frac{1}{2} \int_{\Omega}|v(x)|^{2} d x \\
& -\frac{1}{2_{s}^{*}} \int_{\Omega}|v(x)|^{2_{s}^{*}} d x-\int_{\Omega} G(x, v(x)) d x,
\end{aligned}
$$

where the space $V_{0}$ will be introduced in Sect. 2 .

Along the present paper, $\left\{\mu_{j}\right\}_{j \in \mathbb{N}}$ represents the eigenvalues of the following homogeneous Dirichlet problem:

$$
\begin{cases}-\mathcal{L}_{K} v=\mu \nu & \text { in } \Omega, \\ v=0 & \text { in } \mathbb{R}^{N} \backslash \Omega .\end{cases}
$$

with

$$
\begin{gathered}
0<\mu_{1}<\mu_{2} \leq \cdots \leq \mu_{j} \leq \mu_{j+1} \leq \cdots, \\
\mu_{j} \rightarrow+\infty \text { as } j \rightarrow+\infty
\end{gathered}
$$

and $\left\{f_{j}\right\}$ is the eigenfunction of the eigenvalue $\mu_{j}$. Moreover, we choose $\left\{f_{j}\right\}_{j \in N}$ to standardize, so that this sequence is an orthonormal basis for space $L^{2}(\Omega)$ and an orthogonal basis of $V_{0}$. For a detailed study of the spectrum of operators $-\mathcal{L}_{K}$, please refer to reference [22, Proposition 9 and Appendix A].

Definition 1 In the end, we claim that the eigenvalue $\mu_{j}, j \geq 2$, has multiplicity $k \in \mathbb{N}$ if

$$
\mu_{j-1}<\mu_{j}=\cdots=\mu_{j+k-1}<\mu_{j+k}
$$

In this case, the space composed of the eigenfunctions corresponding to the eigenvalue $\mu_{j}$ is

$$
\operatorname{span}\left\{f_{j}, \ldots, f_{j+k-1}\right\} .
$$


Next, we also give the definition of the best fractional critical Sobolev space constant $S_{K}$ :

$$
S_{K}:=\inf _{v \in V_{0} \backslash\{0\}} \frac{\int_{\mathbb{R}^{2 N}}|v(x)-v(y)|^{2} K(x-y) d x d y}{\left(\int_{\Omega}|v(x)|^{2 *} d x\right)^{\frac{2}{2_{s}^{*}}}} .
$$

The main feature of this paper is that we are concerned about the existence of multiple solutions to the problem (E). To be precise, our main results are as follows.

Theorem 1.1 Set $s \in(0,1), N>2 s, \Omega$ is an open bounded domain in $\mathbb{R}^{N}$ with continuous boundary, and take $K: \mathbb{R}^{N} \backslash\{0\} \rightarrow(0, \infty)$ be a function fulfilling condition (1.3). Let $\mu \in \mathbb{R}$ and take $\mu^{*}$ be the eigenvalue of problem (1.5) given by

$$
\mu^{*}:=\min \left\{\mu_{j}: \mu<\mu_{j}\right\}
$$

and take $k \in \mathbb{N}$ be its multiplicity. Suppose that

$$
\mu \in\left(\mu^{*}-S_{K}|\Omega|^{-\frac{2 s}{N}}, \mu^{*}\right),
$$

where $|\Omega|$ indicates the Lebesgue measure of the set $\Omega$, and $S_{K}$ is defined in (1.7). If $\left(g_{1}\right)-\left(g_{5}\right)$ hold. Then problem (E) has at least $k$ pairs of nontrivial solutions $\left\{-v_{\mu, i}, v_{\mu, i}\right\}$ such that

$$
\left\|v_{\mu, i}\right\|_{V_{0}} \rightarrow 0 \text { as } \mu \rightarrow \mu^{*}
$$

for any $i=1, \ldots, k$.

Remark 1.1 Finding weak solutions to problem (E) is essentially looking for critical points for the functional $J_{K, \mu}(v)$ associated with this equation. In this theorem, we show that in the proper left neighborhood of any eigenvalue of operator $-\mathcal{L}_{k}$ (with homogeneous Dirichlet boundary condition), the number of nontrivial solutions to problem (E) is twice as many as the eigenvalue multiplicity. Our method is based on the abstract critical point theorem thanks to Bartolo, Benci and Fortunato in [7, Theorem 2.4], which will be introduced in Sect. 2. Our result is a generalization of the conclusion in [18].

Remark 1.2 Now, take $\mu^{*}$ be as in (1.8). Hence,

$$
\mu^{*}=\mu_{j} \quad \text { for some } j \in \mathbb{N} \text {. }
$$

Because $\mu^{*}$ has multiplicity $k \in \mathbb{N}$ thanks to assumption, we get

$$
\begin{aligned}
& \mu^{*}=\mu_{1}<\mu_{2}, \quad \text { if } j=1, \\
& \mu_{j-1}<\mu^{*}=\mu_{k}=\cdots=\mu_{j+k-1}<\mu_{j+k}, \quad \text { if } k \geq 2 .
\end{aligned}
$$

We claim that the parameter $\mu$ is a positive constant. Indeed, in view of definition of $\mu^{*}$ and consider (1.6), it is clearly seen that

$$
\mu^{*} \geq \mu_{1}
$$


Furthermore, the variational characterization of the first eigenvalue $\mu_{1}$ (refer to [22, Proposition 9 and Appendix A]) shows that

$$
\mu_{1}=\min _{v \in V_{0} \backslash\{0\}} \frac{\int_{\mathbb{R}^{2 N}}|v(x)-v(y)|^{2} K(x-y) d x d y}{\int_{\Omega}|v(x)|^{2} d x} .
$$

Since it follows from the Hölder inequality that

$$
\int_{\Omega}|v(x)|^{2} d x \leq|\Omega|^{2 s / N}\left(\int_{\Omega}|v(x)|^{2_{s}^{*}} d x\right)^{2 / 2_{s}^{*}}
$$

by the above inequality and (1.12), we have

$$
\mu_{1} \geq S_{K}|\Omega|^{-\frac{2 s}{N}}
$$

which combined with (1.11) yields

$$
\mu^{*} \geq S_{K}|\Omega|^{-\frac{2 s}{N}}
$$

Thus, according to (1.9) and (1.13), we see that the parameter $\mu>0$.

The paper is structured as follows. In Sect. 2 we introduce our workspace and some lemmas that we will use. In Sect. 3 we verify the geometric structure and conditions of the abstract critical point theory, and give me the proof of Theorem 1.1.

\section{Workspace and some lemmas}

In this section, we first introduce our workspace, then present the main tool of this article, and finally provide some basic lemmas to be used in this article.

Problem (E) has variational characteristics. The space for finding weak solutions is functional space $V_{0}$, which is defined as follows (we can refer to [23] for the detailed nature and introduction of this space).

Through this paper, the space $V$ is a linear space of Lebesgue measurable functions from $\mathbb{R}^{N}$ to $\mathbb{R}$ such that the restriction to $\Omega$ of any function $v$ in $V$ belongs to $L^{2}(\Omega)$ and

$$
\begin{aligned}
& \text { the map }(x, y) \mapsto(v(x)-v(y)) \sqrt{K(x-y)} \\
& \qquad \text { is in } L^{2}\left(\left(\mathbb{R}^{N} \times \mathbb{R}^{N}\right) \backslash(\mathfrak{C} \Omega \times \mathfrak{C} \Omega) d x d y\right),
\end{aligned}
$$

where $\mathfrak{C} \Omega:=\mathbb{R}^{N} \backslash \Omega$, and

$$
V_{0}=\left\{v \in V: v(x)=0 \text { a.e. in } \mathbb{R}^{N} \backslash \Omega\right\} .
$$

We recall that the space $V_{0}$ and $V$ are non-empty thanks to $C_{0}^{2} \subseteq V_{0}$ (see [24, Lemma 5.1]): here we need condition (1.3).

The space $V$ is endowed the following norm:

$$
\|v\|_{V}=\|v\|_{L^{2}(\Omega)}+\left(\int_{\mathcal{Z}}|v(x)-v(y)|^{2} K(x-y) d x d y\right)^{\frac{1}{2}}
$$


where $\mathcal{Z}:=\left(\mathbb{R}^{N} \times \mathbb{R}^{N}\right) \backslash \mathcal{Q}$ and $\mathcal{Q}=(\mathfrak{C} \Omega) \times(\mathfrak{C} \Omega) \subset \mathbb{R}^{N} \times \mathbb{R}^{N}$. Furthermore, according to [23, Lemma 6] as a norm on $V_{0}$ we can let the function

$$
V_{0} \ni v \mapsto\|v\|_{V_{0}}=\left(\int_{\mathbb{R}^{2 N}}|v(x)-v(y)|^{2} K(x-y) d x d y\right)^{\frac{1}{2}}
$$

Moreover, $\left(V_{0},\|\cdot\|_{V_{0}}\right)$ is a Hilbert space (see [23, Lemma 7]) with the scalar product given by

$$
V_{0} \times V_{0} \ni(v, \psi) \mapsto\langle v, \psi\rangle_{V_{0}}:=\int_{\mathbb{R}^{2 N}}(v(x)-v(y))(\psi(x)-\psi(y)) K(x-y) d x d y .
$$

We denote the usual fractional Sobolev space by $H^{s}(\Omega)$, which endowed with norm (the so-called Gagliardo norm) as follows:

$$
\|v\|_{H^{s}(\Omega)}=\|v\|_{L^{2}(\Omega)}+\left(\int_{\Omega \times \Omega}|v(x)-v(y)|^{2}|x-y|^{-(N+2 s)} d x d y\right)^{\frac{1}{2}} .
$$

We note that the norms in (2.1) and (2.4) are not the same when $K(x)=\frac{1}{|x|^{N+2 S}}$, due to $\Omega \times \Omega$ is contained strictly in $\mathcal{Z}$. It makes the space $V_{0}$ different from the usual classical fractional Sobolev space. Thus, from the point of view of the variational method, the classical fractional Sobolev space is insufficient for investigating our problem.

Next, we give the abstract critical point theorem [25, Theorem 2.4].

Theorem 2.1 Let $V_{0}$ be a real Hilbert space with norm $\|\cdot\|$ and assume that $J \in C^{1}\left(V_{0}, \mathbb{R}\right)$ with $J(0)=0$ and $J(v)=J(-v)$, also satisfying the following conditions:

$\left(J_{1}\right)$ there exists $\alpha>0$ such that the Palais-Smale condition for J holds in $(0, \alpha)$;

$\left(J_{2}\right)$ there exist two closed subspaces $X, Y \subset V_{0}$ and positive constants $\varrho, \sigma, \alpha^{\prime}$, with $\sigma<$ $\alpha^{\prime}<\alpha$, such that

(i) $J(v) \leq \alpha^{\prime}$ for any $v \in Y$,

(ii) $J(v) \geq \sigma$ for any $v \in X$ with $\|v\|=\varrho$,

(iii) $\operatorname{codim} X<+\infty$ and $\operatorname{dim} Y \geq \operatorname{codim} X$.

Then there exist at least dim $Y$-codim $X$ pairs of critical points of J, with critical values belonging to the interval $\left[\sigma, \alpha^{\prime}\right]$.

Making use of the behavior of $g$ at zero and at infinity, we give some estimates on the non-linear term $g$ and its primitive $G$ (with respect to its second variable).

Lemma 2.1 ([6, Lemma 6]) Suppose that $g$ is a Carathéodory function satisfying conditions $\left(g_{1}\right)-\left(g_{3}\right)$. Then, for any $\varepsilon>0$ there exists $\delta=\delta(\varepsilon)$ such that a.e. $x \in \Omega$ and for any $t \in \mathbb{R}$

$$
|g(x, t)| \leq 2 \varepsilon|t|+2 \delta(\varepsilon)|t|^{2_{s}^{*}-1},
$$

and so, as a consequence,

$$
|G(x, t)| \leq \varepsilon|t|^{2}+\delta(\varepsilon)|t|^{2_{s}^{*}},
$$

where $G$ is defined as in $\left(g_{4}\right)$. 
The following lemma will be used when we verify the functional geometry.

Lemma 2.2 ([23, Lemma 6]) Let $K: \mathbb{R}^{N} \backslash\{0\} \rightarrow(0, \infty)$ be a function fulfilling condition (1.3). Then there exists a positive constant $d$, depending only on $N$ and s, such that for any $v \in V_{0}$

$$
\|v\|_{L^{2 *}(\Omega)}^{2} \leq d \int_{\mathbb{R}^{2 N}}|v(x)-v(y)|^{2}|x-y|^{-(N+2 s)} d x d y,
$$

where $2_{s}^{*}$ is given in (1.2).

\section{Proof of the main result}

In this section, the idea is to apply Theorem 2.1 to prove that functional satisfies compactness conditions and suitable geometric. It is easy to see that $J_{K, \mu}$ is well defined thanks to [18]. Furthermore, $J_{K, \mu} \in\left(V_{0}, \mathbb{R}\right)$ and

$$
\begin{aligned}
\left\langle J_{K, \mu}^{\prime}(v), \psi\right\rangle= & \langle v, \psi\rangle_{V_{0}}-\mu \int_{\Omega} v(x) \psi d x \\
& -\int_{\Omega}|v(x)|^{2_{s}^{*}-2} v(x) \psi d x-\int_{\Omega} g(x, v(x)) v(x) \psi d x
\end{aligned}
$$

for any $v, \psi \in V_{0}$. Therefore, critical points of functional $J_{K, \mu}$ are solutions to problem (E), it is also the weak solutions of problem (1.4). Observe that the functional $J_{K, \mu}$ is even and functional $J_{K, \mu}(0)=0$ thanks to $\left(g_{5}\right)$. Next, it is suffice to show that the functional $J_{K, \mu}$ fulfills conditions $\left(J_{1}\right)$ and $\left(J_{2}\right)$. In order to this aim, let us do it step by step.

Step 1 (Compactness condition of $J_{K, \mu}$ ). Similar to the proof in the literature [6, Proposition 2], we obtained the following lemma, which states that the functional satisfies the (PS) condition at any level $c$, provided $c$ belongs to a suitable threshold depending on the best fractional critical constant $S_{K}$.

Lemma 3.1 Assume that $g$ is a Carathéodory function satisfying conditions $\left(g_{1}\right)-\left(g_{4}\right)$ and $K: \mathbb{R}^{N} \backslash\{0\} \rightarrow(0, \infty)$ is a function fulfilling condition (1.3). Set $c \in \mathbb{R}$ such that

$$
c<\frac{s}{N} S_{K}^{\frac{N}{2 s}}
$$

and take $v_{j}$ a sequence in $V_{0}$ such that

$$
J_{K, \mu}\left(v_{j}\right) \rightarrow c, \quad \sup _{\|\psi\|_{V_{0}=1}}\left\{\left|\left\langle J_{K, \mu}^{\prime}\left(v_{j}\right), \psi\right\rangle\right|\right\} \rightarrow 0 \quad \text { as } j \rightarrow \infty
$$

Then there exists $v_{\infty} \in V_{0}$ such that, up to a subsequence, $\left\|v_{j}-v_{\infty}\right\|_{V_{0}} \rightarrow 0$ as $j \rightarrow+\infty$.

Remark 3.1 Since our condition $\left(g_{4}\right)$ can imply the (1.11) and (1.12) conditions in Ref. [18], hence we can prove our lemma as Ref. [6, Proposition 2], which is omitted here.

Step 2 (Geometric stucture of $J_{K, \mu}$ ). Using the notation in Theorem 2.1, we take

$$
Y=\operatorname{span}\left\{f_{1}, \ldots, f_{j+k-1}\right\}
$$


and

$$
X= \begin{cases}V_{0} & \text { if } j=1 \\ \left\{v \in V_{0}:\left\langle v, f_{i}\right\rangle_{V_{0}}=0 \forall i=1, \ldots, j-1\right\} & \text { if } j \geq 2\end{cases}
$$

Obviously both $X$ and $Y$ are closed subspaces of $V_{0}$ and

$$
\operatorname{dim} Y=j+k-1, \quad \operatorname{codim} X=j-1
$$

Hence, we see that $\left(J_{2}\right)$ (iii) of Theorem 2.1 is satisfied.

Next, let us prove that the functional $J_{K, \mu}$ possesses the geometric properties of Theorem 2.1, that is, it satisfies the conditions $\left(J_{2}\right)(\mathrm{i})$ and (ii) of Theorem 2.1.

Proof Take $v \in Y$. Then

$$
v(x)=\sum_{i=1}^{j+k-1} v_{i} f_{i}(x)
$$

where $v_{i} \in \mathbb{R}, i=1, \ldots, j+k-1$. Because $\left\{f_{i}, \ldots, f_{j}, \ldots\right\}$ is an orthonormal basis of $L^{2}(\Omega)$ and an orthogonal basis of $V_{0}$, considering (1.10) we have

$$
\|v\|_{V_{0}}^{2}=\sum_{i=1}^{j+k-1} v_{i}^{2}\left\|f_{i}\right\|_{V_{0}}^{2}=\sum_{i=1}^{j+k-1} \mu_{i} v_{i}^{2} \leq \mu_{j} \sum_{i=1}^{j+k-1} v_{i}^{2}=\mu_{j}\|v\|_{L^{2}(\Omega)}^{2}=\mu^{*}\|v\|_{L^{2}(\Omega)}^{2},
$$

hence, according to this and Hölder inequality, $\left(g_{4}\right)$, we get

$$
\begin{aligned}
J_{K, \mu}(v)= & \frac{1}{2} \int_{\mathbb{R}^{2 N}}|v(x)-v(y)|^{2} K(x-y) d x d y-\mu \frac{1}{2} \int_{\Omega}|v(x)|^{2} d x \\
& -\frac{1}{2_{s}^{*}} \int_{\Omega}|v(x)|^{2_{s}^{*}} d x-\int_{\Omega} G(x, v(x)) d x \\
\leq & \frac{1}{2}\left(\mu^{*}-\mu\right) \int_{\Omega}|v(x)|^{2} d x-\frac{1}{2_{s}^{*}} \int_{\Omega}|v(x)|^{2_{s}^{*}} d x \\
\leq & \frac{1}{2}\left(\mu^{*}-\mu\right)|\Omega|^{\frac{2 s}{N}}\left(\int_{\Omega}|v(x)|^{2_{s}^{*}} d x\right)^{\frac{2}{2_{s}^{*}}}-\frac{1}{2_{s}^{*}} \int_{\Omega}|v(x)|^{2_{s}^{*}} d x,
\end{aligned}
$$

thanks to $\left(g_{4}\right)$. Now, for $m \geq 0$ take

$$
h(m)=\frac{1}{2}\left(\mu^{*}-\mu\right)|\Omega|^{\frac{2 s}{N}} m^{2}-\frac{1}{2_{s}^{*}} m^{2^{*}} .
$$

Obverse that $h$ is differentiable in $(0,+\infty)$ and

$$
h^{\prime}(m)=\left(\mu^{*}-\mu\right)|\Omega|^{\frac{2 s}{N}} m-m^{2 *-1}
$$

as a result $h^{\prime}(m) \geq 0$ if and only if

$$
m \leq \bar{m}=\left(\left(\mu^{*}-\mu\right)|\Omega|^{\frac{2 s}{N}}\right)^{\frac{1}{2_{s}^{*}-2}} .
$$


Thus, the function $h$ maximizes at $\bar{m}$, and for any $m \geq 0$

$$
h(m) \leq \max _{m \geq 0} h(m)=h(\bar{m})=\frac{s}{N}\left(\mu^{*}-\mu\right)^{\frac{N}{2 s}}|\Omega| .
$$

It follows from (3.2) and (3.3) that

$$
\sup _{v \in Y} J_{K, \mu}(v) \leq \max _{m \geq 0} h(m)=\frac{s}{N}\left(\mu^{*}-\mu\right)^{\frac{N}{2 s}}|\Omega| .
$$

We note that

$$
\frac{s}{N}\left(\mu^{*}-\mu\right)^{\frac{N}{2 s}}|\Omega|>0
$$

thanks to $\mu<\mu^{*}$ by (1.9).

In the end, take $v \in X$. We claim that

$$
\|v\|_{V_{0}}^{2} \geq \mu^{*}\|v\|_{L^{2}(\Omega)}^{2}
$$

Indeed, If $v$ is equal to 0 , this is obviously true, while if $v \in X \backslash\{0\}$ it follows from the variational characterization of $\mu^{*}=\mu_{j}$ defined by

$$
\mu_{j}=\min _{\nu \in V_{0} \backslash\{0\}} \frac{\int_{\mathbb{R}^{2 N}}|v(x)-v(y)|^{2} K(x-y) d x d y}{\int_{\Omega}|v(x)|^{2} d x},
$$

as proved in [15, Proposition 9].

Therefore, according to Lemma 2.1, Lemma 2.2, Hölder inequality, (1.3) and (3.5), we obtain for any $\varepsilon>0$

$$
\begin{aligned}
& J_{K, \mu}(v)=\frac{1}{2} \int_{\mathbb{R}^{2 N}}|v(x)-v(y)|^{2} K(x-y) d x d y-\mu \frac{1}{2} \int_{\Omega}|v(x)|^{2} d x \\
& -\frac{1}{2_{s}^{*}} \int_{\Omega}|v(x)|^{2_{s}^{*}} d x-\int_{\Omega} G(x, v(x)) d x \\
& \geq \frac{1}{2} \int_{\mathbb{R}^{2 N}}|v(x)-v(y)|^{2} K(x-y) d x d y-\mu \frac{1}{2} \int_{\Omega}|v(x)|^{2} d x-\frac{1}{2_{s}^{*}} \int_{\Omega}|v(x)|^{2_{s}^{*}} d x \\
& -\varepsilon \int_{\Omega}|v(x)|^{2} d x-\delta(\varepsilon) \int_{\Omega}|v(x)|^{2_{s}^{*}} d x \\
& \geq \frac{1}{2}\left(1-\frac{\mu}{\mu^{*}}\right)\|v\|_{V_{0}}^{2}-\varepsilon\|v\|_{L^{2}(\Omega)}^{2}-\left(\frac{1}{2_{s}^{*}}+\delta(\varepsilon)\right)\|v\|_{L_{s}^{2 *}(\Omega)}^{2^{*}} \\
& \geq \frac{1}{2}\left(1-\frac{\mu}{\mu^{*}}\right)\|v\|_{V_{0}}^{2}-\varepsilon|\Omega|^{\frac{2_{s}^{*}-2}{2_{s}^{*}}}\|v\|_{L^{2_{s}^{*}(\Omega)}}^{2}-\left(\frac{1}{2_{s}^{*}}+\delta(\varepsilon)\right)\|v\|_{L_{s}^{2_{s}^{*}}(\Omega)}^{2^{*}} \\
& \geq \frac{1}{2}\left(1-\frac{\mu}{\mu^{*}}\right)\|v\|_{V_{0}}^{2}-\varepsilon d|\Omega|^{\frac{2_{s}^{*}-2}{2_{s}^{*}}} \int_{\mathbb{R}^{N}} \frac{|v(x)-v(y)|^{2}}{|x-y|^{N+2 s}} d x d y \\
& -\left(\frac{1}{2_{s}^{*}}+\delta(\varepsilon)\right) d^{\frac{2 *}{2}}\left(\int_{\mathbb{R}^{N}} \frac{|v(x)-v(y)|^{2}}{|x-y|^{N+2 s}} d x d y\right)^{\frac{2_{s}^{*}}{2}} \\
& \geq\left(\frac{1}{2}\left(1-\frac{\mu}{\mu^{*}}\right)-\frac{\varepsilon d|\Omega|^{\frac{2_{s}^{*}-2}{2_{s}^{*}}}}{\vartheta}\right)\|v\|_{V_{0}}^{2}-\left(\frac{1}{2_{s}^{*}}+\delta(\varepsilon)\right)\left(\frac{d}{\vartheta}\right)^{\frac{2_{s}^{*}}{2}}\|v\|_{V_{0}}^{2^{*}}
\end{aligned}
$$




$$
=\|v\|_{V_{0}}^{2}\left(\frac{1}{2}\left(1-\frac{\mu}{\mu^{*}}\right)-\frac{\varepsilon d|\Omega|^{\frac{2_{s}^{*}-2}{2_{s}^{*}}}}{\vartheta}-\left(\frac{1}{2_{s}^{*}}+\delta(\varepsilon)\right)\left(\frac{d}{\vartheta}\right)^{\frac{2_{s}^{*}}{2}}\|v\|_{V_{0}}^{2_{s}^{*}-2}\right),
$$

where $\vartheta$ was defined in (1.3). We take $v \in X$ be such that $\|v\|_{V_{0}}=\varrho>0$. We can choose $\varepsilon$ such that $2 \varepsilon d|\Omega|^{\frac{2_{s}^{*}-2}{22_{s}^{*}}}<\vartheta\left(1-\frac{\mu}{\mu^{*}}\right)$, and let $\varrho$ be small enough, say $\varrho \leq \bar{\varrho}$ with $\varrho>0$, so that

$$
\frac{1}{2}\left(1-\frac{\mu}{\mu^{*}}\right)-\frac{\varepsilon d|\Omega|^{\frac{2_{s}^{*}-2}{2 *}}}{\vartheta}-\left(\frac{1}{2_{s}^{*}}+\delta(\varepsilon)\right)\left(\frac{d}{\vartheta}\right)^{\frac{2_{s}^{*}}{2}} \varrho^{2_{s}^{*}-2}>0
$$

and

$$
\begin{aligned}
& \varrho^{2}\left(\frac{1}{2}\left(1-\frac{\mu}{\mu^{*}}\right)-\frac{\varepsilon d|\Omega|^{\frac{2_{s}^{*}-2}{2 *}}}{\vartheta}-\left(\frac{1}{2_{s}^{*}}+\delta(\varepsilon)\right)\left(\frac{d}{\vartheta}\right)^{\frac{2_{s}^{*}}{2}} \varrho^{2_{s}^{*}-2}\right) \\
& <\frac{\varrho^{2}}{2}\left(1-\frac{\mu}{\mu^{*}}\right)<\frac{s}{N}\left(\mu^{*}-\mu\right)^{\frac{N}{2 s}}|\Omega|
\end{aligned}
$$

thanks to the fact that $2_{s}^{*}>2$.

Proof of Theorem 1.1 From Step 1, it is easy to see that $J_{K, \mu}$ fulfills $\left(J_{1}\right)$ with

$$
\alpha=\frac{s}{N} S_{K}^{\frac{N}{2 s}}>0
$$

Moreover, according to Step 2, it follows from (3.3)-(3.8) that $J_{K, \mu}$ satisfies $\left(J_{2}\right)$ with

$$
\begin{aligned}
& \varrho=\bar{\varrho}, \\
& \alpha^{\prime}=\frac{s}{N}\left(\mu^{*}-\mu\right)^{\frac{N}{2 s}}|\Omega|,
\end{aligned}
$$

and

$$
\sigma=\bar{\varrho}^{2}\left(\frac{1}{2}\left(1-\frac{\mu}{\mu^{*}}\right)-\frac{\varepsilon d|\Omega|^{\frac{2_{s}^{*}-2}{2 *}}}{\vartheta}-\left(\frac{1}{2_{s}^{*}}+\delta(\varepsilon)\right)\left(\frac{d}{\vartheta}\right)^{\frac{2_{s}^{*}}{2}} \varrho^{2_{s}^{*}-2}\right) .
$$

By (3.7), (3.8) and assumption (1.9), we note that

$$
0<\sigma<\alpha^{\prime}<\alpha .
$$

In conclusion, functional $J_{K, \mu}$ satisfies all the conditions in abstract critical point theory (i.e. Theorem 2.1), so the functional has $k$ pairs $\left\{-v_{\mu, i}, v_{\mu, i}\right\}$ of critical points whose values are such that

$$
0<\sigma \leq J_{K, \mu}\left( \pm v_{\mu, i}\right) \leq \alpha
$$

for any $i=1, \ldots, k$.

Furthermore, by (3.9) and $J_{K, \mu}(0)=0$, clearly, these critical points are nontrivial. Therefore, problem (E) has at least $k$ pairs of nontrivial weak solutions $\left\{-v_{\mu, i}, v_{\mu, i}\right\}$. At present, 
fix $i \in\{1, \ldots, k\}$. According to (3.9) and $\left(g_{4}\right)$ we get

$$
\begin{aligned}
\alpha & =\frac{s}{N}\left(\mu^{*}-\mu\right)^{\frac{N}{2 s}}|\Omega| \geq J_{K, \mu}\left(v_{\mu, i}\right) \\
& =J_{K, \mu}\left(v_{\mu, i}\right)-\frac{1}{2}\left\langle J_{K, \mu}^{\prime}\left(v_{\mu, i}\right), v_{\mu, i}\right\rangle \\
& =\left(\frac{1}{2}-\frac{1}{2_{s}^{*}}\right)\left\|v_{\mu, i}\right\|_{L_{s}^{2 *}(\Omega)}^{2^{*}}-\int_{\Omega} G\left(x, v_{\mu, i}(x)\right) d x+\int_{\Omega} \frac{1}{2} g\left(x, v_{\mu, i}(x)\right) v_{\mu, i}(x) d x \\
& \geq \frac{\epsilon}{N}\left\|v_{\mu, i}\right\|_{L^{2}(\Omega)}^{2^{*}} .
\end{aligned}
$$

As a result, passing to the limit as $\mu \rightarrow \mu^{*}$ in (3.10), we obtain

$$
\left\|v_{\mu, i}\right\|_{L_{s}^{2 *}(\Omega)}^{2^{*}} \rightarrow 0 \quad \text { as } \mu \rightarrow \mu^{*}
$$

Hence, by (3.11), since $L^{2 *}(\Omega) \hookrightarrow L^{2}(\Omega)$ is continuous and $\Omega$ is bounded, we also have

$$
\left\|v_{\mu, i}\right\|_{L^{2}(\Omega)}^{2} \rightarrow 0 \quad \text { as } \mu \rightarrow \mu^{*}
$$

So, based on the discussion above and Lemma 2.1, we get

$$
\begin{aligned}
\frac{s}{N}\left(\mu^{*}-\mu\right)^{\frac{N}{2 s}}|\Omega| & \geq J_{K, \mu}\left(v_{\mu, i}\right) \\
& \geq \frac{1}{2}\left\|v_{\mu, i}\right\|_{V_{0}}^{2}-\left(\frac{\mu}{2}+\varepsilon\right)\left\|v_{\mu, i}\right\|_{L^{2}(\Omega)}^{2}-\left(\frac{1}{2_{s}^{*}}+\delta(\varepsilon)\right)\left\|v_{\mu, i}\right\|_{L_{s}^{2 *}(\Omega)}^{2^{*}},
\end{aligned}
$$

which combined with (3.11) and (3.11) implies

$$
\left\|v_{\mu, i}\right\|_{V_{0}} \rightarrow 0 \quad \text { as } \mu \rightarrow \mu^{*}
$$

This means that the proof of Theorem 1.1 is complete.

\section{The prospect of future problems}

In this section we want to generalize our problem to the more general case $p$. The equation that we deal with becomes

$$
-\mathcal{L}_{K}^{p} v=\mu|v|^{p-2} v+|v|^{p_{s}^{*}-2} v+g(x, v) \quad \text { in } \Omega, \quad v=0 \quad \text { in } \mathbb{R}^{N} \backslash \Omega,
$$

where $\Omega$ is an open and bounded domain with Lipschitz boundary, $N>p s$, with $s \in(0,1)$, and $\mu$ is a positive real parameter, $p_{s}^{*}=\frac{p N}{N-p s}$ is the fractional critical Sobolev exponent, while $\mathcal{L}_{K}^{p}$ is an integro-differential operator defined as follows:

$$
\mathcal{L}_{K}^{p} v(x):=2 \lim _{\varepsilon \rightarrow 0^{+}} \int_{\mathbb{R}^{N} \backslash \mathcal{B}_{\varepsilon}(x)}|v(x)-v(y)|^{p-2}(v(x)-v(y)) K(x-y) d y,
$$


for all $x \in \mathbb{R}^{N}$, where $\mathcal{B}_{\varepsilon}(x)=\{z|| x-z \mid<\varepsilon\}$. The function $K: \mathbb{R}^{N} \backslash\{0\} \rightarrow(0, \infty)$ is a kernel such that

$$
\left\{\begin{array}{l}
\omega K \in L^{1}\left(\mathbb{R}^{N}\right) \quad \text { where } \omega(x)=\min \left\{|x|^{p}, 1\right\} ; \\
\text { there exists } \vartheta>0 \text { such that } K(x) \geq \vartheta|x|^{-(N+p s)} \quad \text { for any } x \in \mathbb{R}^{N} \backslash\{0\} \\
K(x)=K(-x) \quad \text { for any } x \in \mathbb{R}^{N} \backslash\{0\} .
\end{array}\right.
$$

Thanks to taking $K(x)=|x|^{-(N+p s)}$, we have $\mathcal{L}_{K}^{p}=-(-\Delta)_{p}^{s}$, so we call the operator $\mathcal{L}_{K}^{p}$ an extension of the fractional $p$-Laplacian.

Similarly, $g$ is a lower order perturbation of the critical power $|v|^{p_{s}^{*}-2} v$, and it is a Carathéodory function from $\Omega \times \mathbb{R}$ to $\mathbb{R}$ satisfying the following assumptions:

$\left(g_{1}^{\prime}\right) \sup \{|g(x, t)|$ : a.e. $x \in \Omega, t \in[0, D]\}<+\infty$ for any $D>0$;

$\left(g_{2}^{\prime}\right) \lim _{|t| \rightarrow 0} \frac{g(x, t)}{t^{p-1}}=0$ uniformly for $x \in \Omega$;

$\left(g_{3}^{\prime}\right) \lim _{|t| \rightarrow \infty} \frac{g(x, t)}{|t|_{s}^{*}-1}=0$ uniformly for $x \in \Omega$;

$\left(g_{4}^{\prime}\right) 0 \leq G(x, t)=\int_{0}^{t} g(x, \widetilde{v}) d \widetilde{s} \leq \frac{1}{p} g(x, t) t+\frac{s-\epsilon}{N}|t|^{p_{s}^{*}}$ a.e. $x \in \Omega, t \in \mathbb{R}$, where $s>\epsilon>0$;

$\left(g_{5}^{\prime}\right) g(x,-t)=-g(x, t)$ for all $x \in \Omega, t \in \mathbb{R}$.

A typical example for $g$ we can set $g(x, t)=l(x)|t|^{\xi-2} t$, with $l(x) \in L^{\infty}(\Omega), l(x) \geq 0$ a.e. $x \in \Omega$ and $\xi \in\left(p, p_{s}^{*}\right)$.

Next, we also give the definition of the best fractional critical Sobolev space constant $S_{K}^{p}$

$$
S_{K}^{p}:=\inf _{v \in V_{0} \backslash\{0\}} \frac{\iint_{\mathbb{R}^{2 N}}|v(x)-v(y)|^{p} K(x-y) d x d y}{\left(\int_{\Omega}|v(x)|^{p_{s}^{*}} d x\right)^{\frac{p}{p_{s}^{*}}}} .
$$

$\left\{\mu_{j}\right\}_{j \in \mathbb{N}}$ represents the eigenvalues of the following problem:

$$
\begin{cases}-\mathcal{L}_{K}^{p} \nu=\mu|v|^{p-2} v & \text { in } \Omega \\ v=0 & \text { in } \mathbb{R}^{\mathbb{N}} \backslash \Omega\end{cases}
$$

with

$$
\begin{gathered}
0<\mu_{1}<\mu_{2} \leq \cdots \leq \mu_{j} \leq \mu_{j+1} \leq \cdots \\
\mu_{j} \rightarrow+\infty \text { as } j \rightarrow+\infty
\end{gathered}
$$

and $\left\{f_{j}\right\}$ is the eigenfunction of the eigenvalue $\mu_{j}$. Moreover, we choose $\left\{f_{j}\right\}_{j \in N}$ to standardize, so that this sequence is an orthonormal basis for space $L^{p}(\Omega)$ and an orthogonal basis of $V_{0}$.

We give the main result of this section.

Theorem 4.1 Set $s \in(0,1), N>p s, \Omega$ is an open bounded domain in $\mathbb{R}^{N}$ with continuous boundary, and take $K: \mathbb{R}^{N} \backslash\{0\} \rightarrow(0, \infty)$ be a function fulfilling condition (4.1). Let $\mu \in \mathbb{R}$ and take $\mu^{*}$ be the eigenvalue of problem (4.4) given by

$$
\mu^{*}:=\min \left\{\mu_{j}: \mu<\mu_{j}\right\}
$$

and take $k \in \mathbb{N}$ be its multiplicity. Suppose that

$$
\mu \in\left(\mu^{*}-S_{K}^{p}|\Omega|^{-\frac{p s}{N}}, \mu^{*}\right),
$$


where $|\Omega|$ indicates the Lebesgue measure of the set $\Omega$, and $S_{K}^{p}$ is defined in (4.3). If $\left(g_{1}^{\prime}\right)-\left(g_{5}^{\prime}\right)$ hold, then problem (4.1) has at least $k$ pairs of nontrivial solutions $\left\{-v_{\mu, i}, v_{\mu, i}\right\}$ such that

$$
\left\|v_{\mu, i}\right\|_{V_{0}} \rightarrow 0 \text { as } \mu \rightarrow \mu^{*}
$$

for any $i=1, \ldots, k$.

Remark 4.1 In this theorem, our workspace $V_{0}$ can be defined in the form in the literature [26]. Our proof method and process are similar to the situation when $p=2$. Therefore, we omit it here. We also show that a multiplicity and bifurcation result for the kind of fractional $p$-Laplacian equation.

\section{Conclusion}

In this work, a multiplicity and bifurcation result for fractional problems is obtained by using the variational and topological methods. Our conclusions improve the related results in some respects. The fractional nonlocal problems with critical nonlinearities have their applications in many fields, for example, in [27] the authors described a model of flow in porous media including nonlocal (long-range) diffusion effects. The model is based on Darcy's law and the pressure is related to the density by an inverse fractional Laplacian operator. They proved the existence of solutions that propagate with finite speed. Examples are flame propagation, water waves, optimization, multiple scattering, thin obstacle problem, image reconstruction, through a new and fascinating scientific approach (see, e.g., the papers $[28,29])$. In the future, such kind of bifurcation results can be obtained in applications to a class of fractional Kirchhoff equations. In this direction, one can refer to Refs. [30-35].

\section{Acknowledgements}

The authors thank the anonymous referees for invaluable comments and insightful suggestions, which improved the presentation of this manuscript.

\section{Funding}

The work is supported by the Fundamental Research Funds for Central Universities (2019B44914), Natural Science Foundation of Jiangsu Province (BK20180500) and the National Key Research and Development Program of China (2018YFC1508100). J. Zuo is also supported by China Scholarship Council (No.201906710004) and Jilin Province

Department of Education "13th five-year plan" Science and technology, research and planning projects.

\section{Availability of data and materials}

Not applicable.

\section{Competing interests}

The authors declare that they have no competing interests.

\section{Authors' contributions}

All authors read and approved the final manuscript and all authors have agreed to the authorship and the order of authorship for this manuscript. All authors have the appropriate permissions and rights to the reported data.

\section{Author details}

${ }^{1}$ Faculty of Applied Sciences, Jilin Engineering Normal University, Changchun, P.R. China. ${ }^{2}$ College of Science, Hohai University, Nanjing, China. ${ }^{3}$ Departamento de Matemática, Universidade Estadual de Campinas, IMECC, Campinas, Brazil. ${ }^{4}$ School of Earth Sciences and Engineering, Hohai University, Nanjing, China. ${ }^{5}$ Biostatistics Department, College of Public Health, University of Georgia Health Science Campus, Athens, USA. ${ }^{6}$ School of Electronic and Information Engineering, Jiangxi Industry Polytechnic College, Nanchang, P.R. China.

\section{Publisher's Note}

Springer Nature remains neutral with regard to jurisdictional claims in published maps and institutional affiliations. 


\section{References}

1. Molica Bisci, G., Servadei, R.: Lower semicontinuity of functionals of fractional type and applications to nonlocal equations with critical Sobolev exponent. Adv. Differ. Equ. $207 / 8,(2015)$

2. Barrios, B., Colorado, E., De Pablo, A., Sánchez, U.: On some critical problems for the fractional Laplacian operator. J. Differ. Equ. 252, 6133-6162 (2012)

3. Tan, J.: The Brezis-Nirenberg type problem involving the square root of the Laplacian. Calc. Var. Partial Differ. Equ. 36(1-2), 21-41 (2011)

4. Servadei, R., Valdinoci, E.: A Brezis-Nirenberg result for non-local critical equations in low dimension. Commun. Pure Appl. Anal. 12(6), 2445-2464 (2013)

5. Servadei, R.: The Yamabe equation in a non-local setting. Adv. Nonlinear Anal. 2, 235-270 (2013)

6. Servadei, R., Valdinoci, E.: The Brezis-Nirenberg result for the fractional Laplacian. Trans. Am. Math. Soc. 367(1), 67-102 (2015)

7. Brezis, H., Nirenberg, L.: Positive solutions of nonlinear elliptic equations involving critical Sobolev exponents. Commun. Pure Appl. Math. 36(4), 437-477 (1983)

8. Capozzi, A., Fortunato, D., Palmieri, G.: An existence result for nonlinear elliptic problems involving critical Sobolev exponent. Ann. Inst. Henri Poincaré, Anal. Non Linéaire 2(6), 463-470 (1985)

9. Devillanova, G., Solimini, S.: A multiplicity result for elliptic equations at critical growth in low dimension. Commun. Contemp. Math. 5(2), 171-177 (2003)

10. Schechter, M., Zou, W.: On the Brezis-Nirenberg problem. Arch. Ration. Mech. Anal. 197(1), 337-356 (2010)

11. Servadei, R.: A critical fractional Laplace equation in the resonant case. Topol. Methods Nonlinear Anal. 43(1), 251-267 (2014)

12. Szulkin, A., Weth, T., Willem, M.: Ground state solutions for a semilinear problem with critical exponent. Differ. Integral Equ. 22(9-10), 913-926 (2009)

13. Cerami, G., Fortunato, D., Struwe, M.: Bifurcation and multiplicity results for nonlinear elliptic problems involving critical Sobolev exponents. Ann. Inst. Henri Poincaré, Anal. Non Linéaire 1(5), 341-350 (1984)

14. Chen, Z., Shioji, N., Zou, W.: Ground state and multiple solutions for a critical exponent problem. Nonlinear Differ. Equ. Appl. 19(3), 253-277 (2012)

15. Devillanova, G., Solimini, S.: Concentration estimates and multiple solutions to elliptic problems at critical growth. Adv. Differ. Equ. 7(10), 1257-1280 (2002)

16. Cerami, G., Solimini, S., Struwe, M.: Some existence results for superlinear elliptic boundary value problems involving critical exponents. J. Funct. Anal. 69(3), 289-306 (1986)

17. Clapp, M., Weth, T.: Multiple solutions for the Brezis-Nirenberg problem. Adv. Differ. Equ. 10(4), $463-480$ (2005)

18. Servadei, R., Valdinoci, E.: Fractional Laplacian equations with critical Sobolev exponent. Rev. Mat. Complut. 28(3)، 655-676 (2015)

19. Di Nezza, E., Palatucci, G., Valdinoci, E.: Hitchhiker's quide to the fractional Sobolev spaces. Bull. Sci. Math. 136(5), 521-573 (2012)

20. Molica Bisci, G., Servadei, R.: A bifurcation result for non-local fractional equations. Anal. Appl. (Singap.) 13, 371-394 (2015)

21. Dwivedi, G., Tyagi, J., Verma, R.B.: On the bifurcation results for fractional Laplace equations. Math. Nachr. 290(16), 2597-2611 (2017)

22. Servadei, R., Valdinoci, E.: Variational methods for non-local operators of elliptic type. Discrete Contin. Dyn. Syst. 33(5), 2105-2137 (2013)

23. Servadei, R., Valdinoci, E.: Mountain pass solutions for non-local elliptic operators. J. Math. Anal. Appl. 389, 887-898 (2012)

24. Servadei, R., Valdinoci, E.: Lewy-Stampacchia type estimates for variational inequalities driven by (non)local operators. Rev. Mat. Iberoam. 29(3), 1091-1126 (2013)

25. Bartolo, P., Benci, V., Fortunato, D.: Abstract critical point theorems and applications to some nonlinear problems with strong resonance at infinity. Nonlinear Anal. 7, 981-1012 (1983)

26. Xiang, M.Q., Zhang, B.L., Massimiliano, F.: Existence of solutions for Kirchhoff type problem involving the non-local fractional $p$-Laplacian. J. Math. Anal. Appl. 424, 1021-1041 (2015)

27. Vlahos, L., Isliker, H., Kominis, Y., Hizonidis, K.: Normal and anomalous diffusion: a tutorial. In: Bountis, T. (ed.) Order and Chaos, 10th Volume. Patras University Press, Greece (2008)

28. Cont, R., Tankov, P.: Financial Modelling with Jump Processes. Financial Mathematics Series. Chapman \& Hall, London (2004)

29. Valdinoci, E.: From the long jump random walk to the fractional Laplacian. Bol. Soc. Esp. Mat. Apl. 49, 33-44 (2009)

30. Tyagi, J.: Eigenvalue problem for fractional Kirchhoff Laplacian. Atti Accad. Naz. Lincei, Rend. Lincei, Mat. Appl. 29(1), 195-203 (2018)

31. do Ó, J.M., He, X., Mishra, P.K.: Fractional Kirchhoff problem with critical indefinite nonlinearity. Math. Nachr. 292(3), 615-632 (2019)

32. Binlin, Z., Fiscella, A., Liang, S.: Infinitely many solutions for critical degenerate Kirchhoff type equations involving the fractional p-Laplacian. Appl. Math. Optim. 80(1), 63-80 (2019)

33. Zuo, J., An, T., Li, M.: Superlinear Kirchhoff-type problems of the fractional $p$-Laplacian without the (AR) condition. Bound. Value Probl. 2018, 180 (2018)

34. Zuo, J., An, T., Ye, G., Qiao, Z.: Nonhomogeneous fractional p-Kirchhoff problems involving a critical nonlinearity. Electron. J. Qual. Theory Differ. Equ. 2019, 41 (2019)

35. Zuo, J., An, T., Ye, G.: A class of critical magnetic fractional Kirchhoff problems. Symmetry 12(1), 76 (2020) 